\title{
Challenges in Intracerebral Hemorrhage Clinical Research?
}

\author{
Reza Behrouz
}

Intracerebral hemorrhage $(\mathrm{ICH})$ is the most devastating form of stroke for which no treatment beyond supportive care exists. Numerous clinical trials over the years have sought to identify therapeutic strategies that would improve the outcomes for victims of ICH. To date, no specific agent or surgical treatment has proven effective in reducing disability associated with this devastating condition.

Many of the studies conducted in the past 5 years have been focused on refining the existing treatment paradigms. Two large trials were conducted to identify the optimal blood pressure target for patient presenting with acute ICH $[1,2]$. The results of the two trials were somewhat contradicting and hence, no clear answer could be derived from them. A phase II trial failed to prove that the iron chelator deferoxamine, used as a neuroprotective agent, improved outcomes in ICH [3]. Tranexamic acid, an antifibrinolytic drug, was investigated in a large randomized placebo-controlled trial [4]. Although there were less hematoma expansions in the treatment group, functional status at 90 days after did not differ significantly between the two arms. In patients with intraventricular hemorrhage and a ventriculostomy drain, irrigation with alteplase did not substantially improve functional outcomes, compared with irrigation with saline [5]. Finally, minimally invasive catheter evacuation of the hematoma followed by thrombolysis failed to improve functional outcomes [6].

There were also studies that changed the treatment paradigms for acute ICH. The Platelet Transfusion in Cerebral Haemorrhage $(\mathrm{PATCH})$ trial investigated whether platelet transfusion with standard care, compared with standard care alone, reduced death or dependence after ICH patients on antiplatelet drugs [7]. Platelet transfusion was not only futile, but it was even found to be harmful. The INCH trial compared the efficacies of fresh frozen plasma and prothrombin complex concentrate in ICH patients using vitamin $\mathrm{K}$ antagonists (Warfarin) [8]. Four-factor prothrombin complex concentrate was superior to fresh frozen plasma with respect to international normalized ratio (INR) normalization. It also normalized INR faster and was associated with smaller hematoma expansion, compared to fresh frozen plasma.

Currently, two clinical studies are starting, both sponsored by the National Institutes of Health. Anticoagulation for

Manuscript submitted January 16, 2020, accepted January 22, 2020

Medical Arts and Research Center, School of Medicine, University of Texas Health Science Center at San Antonio, 700 Floyd Curl Drive, San Antonio, TX 78205, USA. Email: behrouz@uthscsa.edu
Stroke Prevention and Recovery after ICH (ASPIRE) is a randomized, double-blinded, phase 3 clinical trial designed to test the efficacy and safety of apixaban, compared with aspirin, in patients with a recent ICH and high-risk non-valvular atrial fibrillation. Seven hundred patients will be recruited and followed for outcomes over a median of 24 months. The primary aim of ASPIRE is to determine if apixaban is superior to aspirin for prevention of the composite outcome of any stroke (hemorrhagic or ischemic) or death from any cause in patients with recent ICH and atrial fibrillation.

Statins Use in Intracerebral Hemorrhage Patient (SATURN) trial will evaluate the effects of continuation versus discontinuation of statins on the risk of ICH recurrence during 24 months of follow-up in patients presenting with a spontaneous lobar ICH while taking a statin drug. It is a multi-center, prospective, randomized, phase 3, pragmatic, open-label, and blinded end-point assessment clinical trial that intends to recruit 1,456 participants.

Research in the past 5 years has shown that medical and surgical treatments for ICH are elusive. Science is yet to find a single therapeutic strategy that would improve outcomes and lessen the extent of disability in these patients. Another challenge with ICH research is coming up with sound ideas or hypotheses for clinical trials. Consequently, efforts are now primarily focused on re-inventing the wheel, refining what is already known, or validating anecdotal practices in place for years.

As ICH is the most pernicious type of stroke, research on this topic should be prioritized. Any new idea, however, that proposes a therapeutic strategy for ICH should be embraced by funding sources, including the National Institutes of Health and the American Heart Association. It is only by trial and error that we could hopefully find the remedy for this devastating condition.

\section{Acknowledgments}

None to declare.

\section{Financial Disclosure}

The author has received funding from the NIH StrokeNet for ASPIRE and SATURN trials.

\section{Conflict of Interest}

None to declare. 


\section{References}

1. Qureshi AI, Palesch YY, Barsan WG, Hanley DF, Hsu CY, Martin RL, Moy CS, et al. Intensive blood-pressure lowering in patients with acute cerebral hemorrhage. N Engl J Med. 2016;375(11):1033-1043.

2. Anderson CS, Heeley E, Huang Y, Wang J, Stapf C, Delcourt C, Lindley R, et al. Rapid blood-pressure lowering in patients with acute intracerebral hemorrhage. N Engl J Med. 2013;368(25):2355-2365.

3. Selim M, Foster LD, Moy CS, Xi G, Hill MD, Morgenstern LB, Greenberg SM, et al. Deferoxamine mesylate in patients with intracerebral haemorrhage (i-DEF): a multicentre, randomised, placebo-controlled, double-blind phase 2 trial. Lancet Neurol. 2019;18(5):428-438.

4. Sprigg N, Flaherty K, Appleton JP, Al-Shahi Salman R, Bereczki D, Beridze M, Ciccone A, et al. Tranexamic acid to improve functional status in adults with spontaneous intracerebral haemorrhage: the TICH-2 RCT. Health Technol Assess. 2019;23(35):1-48.

5. Hanley DF, Lane K, McBee N, Ziai W, Tuhrim S, Lees KR,
Dawson J, et al. Thrombolytic removal of intraventricular haemorrhage in treatment of severe stroke: results of the randomised, multicentre, multiregion, placebo-controlled CLEAR III trial. Lancet. 2017;389(10069):603-611.

6. Hanley DF, Thompson RE, Rosenblum M, Yenokyan G, Lane K, McBee N, Mayo SW, et al. Efficacy and safety of minimally invasive surgery with thrombolysis in intracerebral haemorrhage evacuation (MISTIE III): a randomised, controlled, open-label, blinded endpoint phase 3 trial. Lancet. 2019;393(10175):1021-1032.

7. Baharoglu MI, Cordonnier C, Al-Shahi Salman R, de Gans K, Koopman MM, Brand A, Majoie CB, et al. Platelet transfusion versus standard care after acute stroke due to spontaneous cerebral haemorrhage associated with antiplatelet therapy (PATCH): a randomised, open-label, phase 3 trial. Lancet. 2016;387(10038):2605-2613.

8. Steiner T, Poli S, Griebe M, Husing J, Hajda J, Freiberger A, Bendszus $M$, et al. Fresh frozen plasma versus prothrombin complex concentrate in patients with intracranial haemorrhage related to vitamin $\mathrm{K}$ antagonists (INCH): a randomised trial. Lancet Neurol. 2016;15(6):566-573. 